\title{
A Truly-Redundant Aerial Manipulator System with Application to Push-and-Slide Inspection in Industrial Plants
}

\author{
Marco Tognon ${ }^{1}$, Hermes A. Tello Chávez ${ }^{1}$, Enrico Gasparin ${ }^{2}$, Quentin Sablé ${ }^{\text {, Davide Bicego }}{ }^{1}$, \\ Anthony Mallet ${ }^{1}$, Marc Lany ${ }^{2}$, Gilles Santi ${ }^{2}$, Bernard Revaz ${ }^{2}$, Juan Cortés ${ }^{1}$, Antonio Franchi ${ }^{1}$
}

\begin{abstract}
We present the design, motion planning and control of an aerial manipulator for non-trivial physical interaction tasks, such as pushing while sliding on curved surfaces - a task which is motivated by the increasing interest in autonomous non-destructive tests for industrial plants. The proposed aerial manipulator consists of a multidirectional-thrust aerial vehicle - to enhance physical interaction capabilities - endowed with a 2-DoFs lightweight arm - to enlarge its workspace. This combination makes it a truly-redundant manipulator going beyond standard aerial manipulators based on collinear multirotor platforms. The controller is based on a PID method with a 'displaced' positional part that ensures asymptotic stability despite the arm elasticity. A kinodynamic task-constrained and control-aware global motion planner is used. Experiments show that the proposed aerial manipulator system, equipped with an Eddy Current probe, is able to scan a metallic pipe sliding the sensor over its surface and preserving the contact. From the measures, a weld on the pipe is successfully detected and mapped.
\end{abstract}

\section{INTRODUCTION}

In industrial facilities, the assessment of the structural integrity is a mandatory process to be performed regularly, especially in sectors like oil\&gas and water industries, where the integrity inspection of low carbon steel welds over pipes is very frequent, since cracks or defects can possibly occur. A very important role is played by Non-Destructive Testing (NDT), since it allows assessing the status of an industrial plant without damaging or altering its parts. Among the available NDT techniques for weld inspection, Eddy-Current (EC) [1] is particularly advantageous because it does not require the preparation of the inspecting surface. Furthermore, it is also used for other applications as, e.g., wall-thinning.

Nowadays, the inspection task is typically conducted by human operators that often have to access dangerous areas (e.g., elevated points) with the use of hazardous equipment like climbing ropes or temporary scaffolds. This aspect led to a growing interest in the development and deployment of structural health monitoring solutions [2] or remotely operated robots. The use of robots also allows generating very useful inspections maps [3]. In fact, the precise location of a weld on a pipe is not always known a priori and is neither easily retrievable with visual sensors, especially when pipes are painted or covered by insulating materials.

${ }^{1}$ LAAS-CNRS, Université de Toulouse, CNRS, Toulouse, France, marco.tognon@laas.fr, antonio.franchi@laas.fr,

${ }^{2}$ SENSIMA INSPECTION, Av. Mont Blanc 31, 1196 Gland, Switzerland www.sensimainsp.com, infolsensimainsp.com

This work was funded by the European Union's Horizon 2020 research and innovation programme under grant agreement No 644271 AEROARMS.

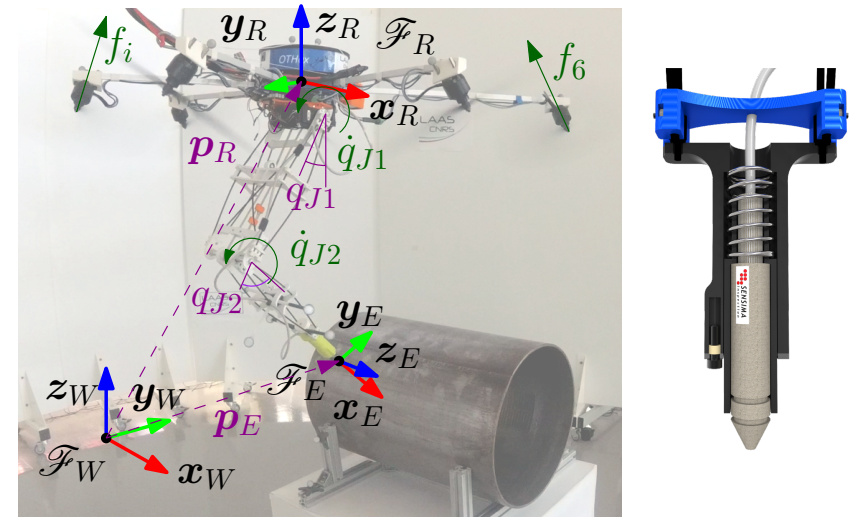

Fig. 1: Aerial manipulator with main variables on the left. On the right a sliced visualization of the compliant sensor holder.

Inspection tasks with robots are challenging because they require an accurate physical interaction in which the probe has to be kept in contact with, and perpendicular to, a curved surface. This contact-based inspection is only one example of the many other applications requiring robots to slide an end-effector on a curved surface while pushing it against the surface, ensuring the contact. Both the pose of the end-effector and the interaction force have to be accurately controlled.

EC inspections of industrial plants with aerial robots is gaining interest in the last years. However, aerial vehicles are still practically employed only for contact-free tasks. This is because of the extremely challenging nature of aerial physical interaction problem, currently under investigation by several research labs and projects like Aeroarms [4]. The aerial systems proposed to face the physical interaction problem range from unidirectional-thrust aerial vehicles endowed with rigid or articulated arms [5], [6], to more recent multidirectional-thrust vehicles [7]. The control methods range from decentralized methods to admittance based methods [7], passing through flatness-based [8] and dynamicinversion-based methods [9]. The majority of these works present methods to enhance aerial interaction capabilities of aerial vehicles, but only a few addressed real physical interaction tasks although for simple vertical flat surfaces, like [10], [11].

The first contribution of this work is to present one of the first complete aerial robotic solutions with sufficient physical interaction capabilities for generic push-and-slide tasks on 
curved surfaces. Such achievement goes substantially beyond simpler tasks such as pick\&place and pull/push objects. The second main contribution is to demonstrate that such a system, endowed with an EC sensor, can successfully inspect a metallic pipe and localize a weld on it. The experiments show that the proposed aerial manipulator is able to autonomously scan the pipe surface sliding the sensor while ensuring the contact and its perpendicularity with respect to (w.r.t.) the surface. From the EC sensor data, the weld is detected and exactly located.

The success of such automatic inspection experiment comes from a wise conception of the aerial manipulator and from the design and integration of state-of-the-artmethods for the EC sensing, motion planning, and control of the robot. Our aerial manipulator is composed of a multidirectional-thrust (i.e., fully actuated) vehicle endowed with a lightweight arm ending with the EC sensor. The system has been designed in order to have a certain redundancy w.r.t. the task, which grants it a greater dexterity. Furthermore, using a multidirectional-thrust vehicle - rather than a unidirectional one - a true redundancy is ensured. In fact, thanks to its full actuation, the vehicle attitude and position, and the exerted 3D force and 3D torque are four independent quantities that can be assigned and controlled to fit at best the task specifications. A kinodynamic task-constrained and control-aware global motion planner is used to generate the robot trajectory for the inspection path, respecting at the same time the system dynamics and input constraints. A simple but effective controller has been designed to let the robot follow the planned trajectory with sufficiently small errors and preserve the contact with the pipe surface, despite the lack of force feedback, the presence of uncertainties (like frictions), and the flexibility of the structure.

The focus of this work is on the integration of design, control, motion planning and sensing system for pipe inspection. For the proof of concept, the experimental validation was conducted indoor using a Motion Capture (MoCap) system. Nevertheless, we degraded the MoCap measurements to emulate the effect of a less accurate localization system.

In section II, we describe the pipe inspection task. The proposed aerial manipulator is presented in Sec. III. The motion planning and control methods are described in Sec. IV and Sec. V, respectively. Experimental results are presented in Sec. VI. A final discussion follows in Sec. VII. Additional details and experimental results are available in a technical report added to the multimedia materials.

\section{PIPE INSPECTION TASK}

The instrument used for EC inspection is a Sensima UPec kit, visible in Fig. 2. The output of the sensor, $w(t) \in \mathbb{C}$ (the field of complex numbers), is related to the properties of the material in contact with the sensor. The trajectory of $w(t)$ allows recognizing features like a crack, variations of the metal composition, etc. For a more detailed and exhaustive explanation, we refer the interested reader to [1]. An example of signal is shown in Fig. 5, where the signal path relative to contact-free flight and weld detection are highlighted.

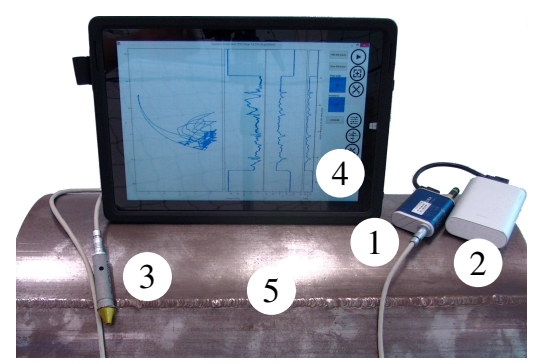

Fig. 2: Measurement setup: The eddy current controller Sensima UPec (1), its battery (2), and the pen-probe (3) are installed on the robot. The measurements are sent through bluetooth to a PC (4) and processed by the software UPecView. We removed the usual coating/paint that insulates the pipe to make the weld (5) visible. In a real scenario the weld would be detectable but not visible.

In particular, from $w$, it is possible to retrieve the magnitude of the signal directly linked to the lift-off and to the weld real signals, denoted by $w_{l}$ and $w_{w}$, respectively. The first is related to the distance from the surface, while the second to the presence or not of a weld. Methods like Principal Component Analysis (PCA) and basis transformation [12] are used to extract those two values. The probe is considered

- in contact with the inspected surface if $w_{l}<\bar{w}_{l}$;

- on the weld if both $w_{l}<\bar{w}_{l}$ and $w_{w}>\bar{w}_{w}$.

A preliminary calibration is conducted to define the acceptance thresholds $\bar{w}_{l}$ and $\bar{w}_{w}$ for $w_{l}$ and $w_{w}$, respectively.

The pipe inspection is conducted performing a raster scan (or any other types of scans) under some important manipulation constraints to ensure the quality of the measurements. According to the probe model used in this activity, the sensor should be maintained 1) perpendicular to the surface, with a maximum deviation of around $10^{\circ}$, and 2) with the sensing tip as close as possible to contact, with an air gap always less than $1[\mathrm{~mm}]$. These two important constraints have to be considered at the motion planning and control levels.

\section{AERIAL MANIPULATOR}

The inspection task requires the robot to control both the position and orientation of the end-effector. Therefore, the robot must have at least 6 Degrees of Freedoms (DoFs). This directly excludes the use of unidirectional-thrust vehicles equipped with a rigid tool, for which only the end-effector position and yaw can be independently controlled [13]. One could endow such an aerial vehicle with an articulated arm with at least 2 DoFs. However, existing control techniques for this type of systems lack of accuracy and robustness w.r.t. disturbances and unknown model parameters.

A recent successful solution for aerial physical interaction is the use of multidirectional-thrust (fully actuated) vehicles, like the one proposed in [14], which are fully actuated in the working region. Such a capability allows the aerial platform exerting a six-dimensional wrench in a decoupled fashion [15], permitting to independently control the position and orientation of the robot, and to balance external forces almost instantaneously and without the need of reorienting the body. In our previous work [7], we showed that a 
multidirectional-thrust vehicle can successfully interact with the environment by means of a rigid tool. However, the input limits restrict the admissible orientation of the platform, and in turn the one of the end-effector. Considering the task described in Sec. II, this limits the area that can be inspected with such robots.

To enlarge the feasible scanning area to the full external surface of a pipe we added a planar 2-DoFs lightweight arm ending with a compliant support for the sensor. An image of the robotic system is shown in Fig. 1. The overall system is characterized by 8 DoFs, which makes it redundant w.r.t. the inspection task. This redundancy can be also exploited during motion planning to choose the best configuration in terms of input feasibility, manipulability, and energy efficiency, and to avoid surrounding obstacles (e.g., see [16]).

\section{A. Multidirectional-thrust Aerial Vehicle: the OTHex}

The Open Tilted Hexarotor (OTHex) is a custom-made aerial robot developed at LAAS-CNRS with the purpose of accomplishing aerial physical interaction tasks. The OTHex, conceived and built in our previous work [17], is composed of six coplanar-center propellers with a tilted arrangement that enables the multidirectional-thrust property and hence the local full actuation of its dynamics. Another interesting feature of this robot is its frontal aperture within the structure frame that increases the workspace of the arm, allowing for frontal and upward operations.

To describe the state of the OTHex, and then of the whole robotic system, we define a world frame $\mathscr{F}_{W}=$ $\left\{O_{W}, \boldsymbol{x}_{W}, \boldsymbol{y}_{W}, \boldsymbol{z}_{W}\right\}$, with arbitrarily placed origin $O_{W}$, and unit axes $\left(\boldsymbol{x}_{W}, \boldsymbol{y}_{W}, \boldsymbol{z}_{W}\right)$ such that $\boldsymbol{z}_{W}$ points in the opposite direction of the gravity vector. An additional frame $\mathscr{F}_{R}=$ $\left\{O_{R}, \boldsymbol{x}_{R}, \boldsymbol{y}_{R}, \boldsymbol{z}_{R}\right\}$ is rigidly attached to the OTHex in its Center of Mass (CoM). The position and orientation of the aerial vehicle are defined by the vector $p_{R} \in \mathbb{R}^{3}$ and the rotation matrix $\boldsymbol{R}_{R} \in S O(3)$, respectively. The linear and angular velocities are defined by the vectors $\boldsymbol{v}_{R}=d \boldsymbol{p}_{R} / d t$ and $\omega_{R} \in \mathbb{R}^{3}$ that is the angular velocity of $\mathscr{F}_{R}$ w.r.t. $\mathscr{F}_{W}$ and expressed in $\mathscr{F}_{R}$. In working conditions, we can assume that the platform is fully actuated, namely capable to independently generate any force $\boldsymbol{f}_{R} \in \mathbb{R}^{3}$ and torque $\tau_{R} \in \mathbb{R}^{3}$, both expressed in $\mathscr{F}_{R}$.

\section{B. Articulated Arm and End-effector}

The 2-DoF serial manipulator presents a tensegrity-based structure built from prestressed carbon fiber bars connected through 3D printed ABS parts. A similar concept was successfully used for aerial manipulation in [18]. The tensegritybased structure leads to the minimization of the mass and inertia of the structure at the expense of the admissible payload and rigidity. To reduce the inertia effects, the two Dynamixel servomotors MX-64T and MX-28T are fixed to the base of the manipulator as close as possible to the CoM of the aerial platform. The actuation of the second joint is ensured by means of a synchronous belt.

To describe the arm configuration, we rigidly attach to each link a frame $\mathscr{F}_{J i}=\left\{\boldsymbol{O}_{J i}, \boldsymbol{x}_{J i}, \boldsymbol{y}_{J i}, \boldsymbol{z}_{J i}\right\}$ with $i=1,2$, fol- lowing the Denavit-Hartenberg convention: $q_{J i} \in \mathbb{R}$ denotes the rotation angle about the axis of actuation $z_{J i}$ of the $i$ th joint. The arm configuration is then given by the vector $\boldsymbol{q}_{A}=\left[\begin{array}{ll}q_{J 1} & q_{J 2}\end{array}\right]^{\top} \in \mathbb{R}^{2}$, and $\boldsymbol{v}_{A}=\dot{\boldsymbol{q}}_{A} \in \mathbb{R}^{2}$ denotes its velocity.

Each joint is not directly driven by a motor, thus the relation between joint and motor velocities, $\boldsymbol{v}_{M}=\left[\begin{array}{ll}v_{M 1} & v_{M 2}\end{array}\right]^{\top} \in$ $\mathbb{R}^{2}$, is given by $\boldsymbol{v}_{A}=\boldsymbol{J}_{A M} \boldsymbol{v}_{M}$ where $\boldsymbol{J}_{A M} \in \mathbb{R}^{2 \times 2}$ is the corresponding invertible Jacobian matrix. We assume that each motor applies a generalized torque $\tau_{M i} \in \mathbb{R}$ about the joint axis $z_{J i}$, for $i=1,2$. The motors are controlled in velocity', so $\tau_{M i}$ cannot be set at will, but it is assigned by a low-level controller based on the actual and desired motor speed, $v_{M i}^{\star}$.

In order to ensure a continuous contact between the sensor and the pipe despite the tolerance in position, the end-effector needs additional compliance. Hence, the sensor is embedded into a 3D printed custom slider, with an internal compression spring (see Fig. 1 on the right). This compliance element was designed to absorb positioning errors of up to $2[\mathrm{~cm}]$ and having interaction forces still feasible for the robotic system.

To describe the pose of the end-effector, we rigidly attach a frame $\mathscr{F}_{E}=\left\{\boldsymbol{O}_{E}, \boldsymbol{x}_{E}, \boldsymbol{y}_{E}, \boldsymbol{z}_{E}\right\}$ to the tip of the sensor. $\boldsymbol{p}_{E} \in$ $\mathbb{R}^{3}$ and ${ }^{W} \boldsymbol{R}_{E} \in S O(3)$ denote the position and orientation of $\mathscr{F}_{E}$ w.r.t. $\mathscr{F}_{W}$, respectively. The main physical parameters of the whole robotic system are reported in the technical report in the multimedia materials.

\section{Dynamic Model}

We denote by $\boldsymbol{q}=\left(\boldsymbol{p}_{R}, \boldsymbol{R}_{R}, \boldsymbol{q}_{A}\right)$ and $\boldsymbol{v}=\left[\begin{array}{lll}\boldsymbol{v}_{R}^{\top} & \boldsymbol{\omega}_{R}^{\top} & \boldsymbol{v}_{A}^{\top}\end{array}\right]^{\top}$ the configuration and the corresponding velocity vector of the aerial manipulator. Its dynamics can be formulated using the Lagrange method in the following form:

$$
\boldsymbol{M}(\boldsymbol{q}) \dot{\boldsymbol{v}}=\boldsymbol{c}(\boldsymbol{q}, \boldsymbol{v})+\boldsymbol{g}(\boldsymbol{q})+\boldsymbol{G}(\boldsymbol{q}) \boldsymbol{u}
$$

where $\boldsymbol{M}(\boldsymbol{q}) \in \mathbb{R}^{(6+2) \times(6+2)}$ is the positive-definite inertia matrix, $\boldsymbol{c}(\boldsymbol{q}, \boldsymbol{v}) \in \mathbb{R}^{(6+2)}$ is the vector collecting the centrifugal and Coriolis forces, $\boldsymbol{g}(\boldsymbol{q}) \in \mathbb{R}^{(6+2)}$ represents the gravitational term, $\boldsymbol{G}(\boldsymbol{q}) \in \mathbb{R}^{(6+2) \times(6+2)}$ is the input allocation matrix, and $\boldsymbol{u}=\left[\begin{array}{llll}\boldsymbol{f}_{R}^{\top} & \boldsymbol{\tau}_{R}^{\top} & \tau_{M 1} & \tau_{M 2}\end{array}\right]^{\top} \in \mathbb{R}^{(6+2)}$ is the vector containing all the inputs of the aerial manipulator. A more detailed derivation can be found in [9].

\section{MOTION PLANNING METHOD}

To properly identify and map a weld, one solution is to perform a raster scan over the surface of the pipe. A taskconstrained motion planner is required to let the end effector obey the specifications described in Sec. II, and also take into account the dynamics of the aerial manipulator, as well as its kinematics and input limits. For these reasons, we use our previously presented Control-Aware Motion Planner [16]. It is a kinodynamic task-constrained motion planner based on a tight combination of motion planning algorithms and control methods, that was customized for fully-actuated aerial manipulators. For the details, we refer the interested reader to [16]. The planner provides the nominal trajectory of each DoF of the aerial manipulator, $\left(\boldsymbol{p}_{R}^{n}(t), \boldsymbol{R}_{R}^{n}(t), \boldsymbol{q}_{A}^{n}(t)\right)$, 
and, as a consequence, the end-effector desired trajectory, $\left(\boldsymbol{p}_{E}^{d}(t), \boldsymbol{R}_{E}^{d}(t)\right)$. Such trajectories respect the task-constraints, the dynamic constraints, and the input limits of the system.

\section{CONTROL METHOD}

The sought push-and-slide task involves the control of both the pose of the end-effector and the interaction force. Simultaneous motion and force control is a well-studied problem in the state of the art of classical grounded manipulator and several established methods have been proposed (see [19] and the citations therein). Some examples are the admittance and the hybrid position/force controllers. However, most of them rely on the dynamic inversion and on a closed loop force action. The latter can be done with an estimation of the external wrench [7] or through a force sensor. The poor knowledge of the dynamic model, the absence of torque controlled motors and the limited payload, make the previous methods not applicable on aerial manipulators. Especially in our case, the servo motors are at best controlled in velocity and the low stiffness of the arm implies deformations that cannot be easily identified and considered in the model. In these cases, where a force feedback is not available, the common approach is to design a compliant behavior of the end-effector and to plan its trajectory slightly "inside" the surface of interest, as for impedance/compliance control [19]. Thanks to the compliance, the position error will make the system slightly push on the surface preserving the contact.

The presented aerial manipulator has three sources of compliance: 1) the PD-based pose control of the aerial vehicle (explained in the following), 2) the natural flexibility of the arm, and 3) the spring in the sensor support. The last was added to absorb most of the interaction force, avoiding to stress the arm too much, thus reducing the risk to break it.

Given the redundancy of the system, the pose control of the end-effector could be done with a standard Inverse Kinematics Control [5]. However, we experimentally noticed that, due to the flexibility of the arm, a full feedback from the end-effector makes the system unstable ${ }^{1}$.

A possible simple solution is to use a joint space control approach in which each actuated DoF independently tracks the nominal joint trajectory provided by the planner. With a perfect knowledge of the kinematic model, a zero tracking error at the joint level would imply a zero tracking error of the end-effector trajectory. However, this is never the case in real conditions. A feedback action w.r.t. the end-effector pose is always needed. Because of the elasticity, the arm can not precisely compensate high frequency errors of the end-effector. Therefore we decided to delegate the feedback action to the OTHex. Since it is fully actuated, it can directly and precisely compensate errors in all the 6 DoFs of the endeffector. The arm is instead controlled in joint space, i.e.:

$$
\boldsymbol{v}_{M}^{\star}=\boldsymbol{v}_{M}^{n}+\boldsymbol{K}_{M}^{P} \boldsymbol{e}_{M}
$$

\footnotetext{
${ }^{1}$ The results of such an experiment are available in the technical report and in the video added to the multimedia materials. An empirical evaluation of the arms flexibility is also available there.
}

where $\boldsymbol{e}_{M}=\boldsymbol{q}_{M}^{n}-\boldsymbol{q}_{M}, \boldsymbol{K}_{M}^{P} \in \mathbb{R}^{2 \times 2}$ is a positive definite matrix. $\boldsymbol{q}_{M}^{n}$ and $\boldsymbol{v}_{M}^{n}$ are computed from the nominal joints angles and velocities, $\left(\boldsymbol{q}_{A}^{n}, \boldsymbol{v}_{A}^{n}\right)$, by inverse kinematics.

Splitting the task into position and orientation of the endeffector, we experimentally noticed that for the sought task, the arm elasticity and the kinematic errors mainly reflect on the position error. Therefore, we decided to perform also the attitude control of the vehicle directly in the joint space:

$$
\tau_{R}=g_{2}(\boldsymbol{q})+\boldsymbol{M}_{22} \dot{\omega}_{R}^{n}+\boldsymbol{K}_{R}^{D} \dot{\boldsymbol{e}}_{R}+\boldsymbol{K}_{R}^{P} \boldsymbol{e}_{R}+\boldsymbol{K}_{R}^{I} \int \boldsymbol{e}_{R} d \tau,
$$

where $\boldsymbol{g}_{2}(\boldsymbol{q})=\boldsymbol{\Delta}_{\mathbf{2}} \boldsymbol{g}(\boldsymbol{q}), \boldsymbol{M}_{22}=\boldsymbol{\Delta}_{\mathbf{2}} \boldsymbol{M}(\boldsymbol{q}) \boldsymbol{\Delta}_{\mathbf{2}}^{\top}$ in which ${ }^{2}$ $\boldsymbol{\Delta}_{\mathbf{2}}=\operatorname{diag}\left(\mathbf{0}_{3}, \boldsymbol{I}_{3}, \mathbf{0}_{2}\right) . \boldsymbol{K}_{\star}^{R} \in \mathbb{R}^{3 \times 3}$ are positive definite matrices. Then, $e_{R}=\frac{1}{2}\left[\boldsymbol{R}_{R}^{\top} \boldsymbol{R}_{R}^{n}-\boldsymbol{R}_{R}^{n \top} \boldsymbol{R}_{R}\right]_{\vee}$ in which $[\star]_{\vee}$ is the inverse of the skew map, $\dot{e}_{R}=\boldsymbol{\omega}_{R}^{n}-\boldsymbol{\omega}_{R}$.

Regarding the position control of the vehicle, it has to include the feedback from the end-effector position. However, the design of such control action has to be done carefully due to the presence of elasticity. In view of this, we naturally took inspiration from the state-of-the-art on control of manipulators with elastic joints [20]. Comparing our situation with a manipulator with elastic joints, we can consider the OTHex as a 6-dimensional 'motor' and the arm plus the compliant sensor holder as a single link connected to the 'motor' through 6D passive elastic joint. Inspired by this analogy, we decided to apply a 'dislocated' PD control law, e.g., a PD with a mixed feedback strategy. In fact, it has been proved that for a 1-DoF manipulator with an elastic joint in the one-dimensional case, a feedback action entirely based on the end-effector position and velocity leads to instability, no matter the gain values. On the other hand, a mixed feedback from the link position and motor velocity ensures stability if the value of the $\mathrm{P}$ (proportional) gain is lower than the stiffness of the mechanical elasticity ${ }^{3}$. The proposed position controller employs such a mixed feedback strategy using the position of the end-effector and the translational velocity of the aerial vehicle in the feedback loop:

$$
\begin{aligned}
\boldsymbol{f}_{R}= & \boldsymbol{R}_{R}^{\top}\left(\boldsymbol{g}_{1}(\boldsymbol{q})+\boldsymbol{M}_{11} \dot{\boldsymbol{v}}_{R}^{n}+\right. \\
& \left.+\boldsymbol{K}_{P}^{D} \dot{\boldsymbol{e}}_{\boldsymbol{p}_{R}}+\boldsymbol{K}_{P}^{P} \boldsymbol{e}_{E}+\boldsymbol{K}_{P}^{I} \boldsymbol{\sigma}\left(\boldsymbol{e}_{E}\right)\right),
\end{aligned}
$$

where $\boldsymbol{g}_{1}(q)=\boldsymbol{\Delta}_{\mathbf{1}} \boldsymbol{g}(\boldsymbol{q}), \boldsymbol{M}_{11}=\boldsymbol{\Delta}_{\mathbf{1}} \boldsymbol{M}(\boldsymbol{q}) \boldsymbol{\Delta}_{1}^{\top}$ in which $\boldsymbol{\Delta}_{\mathbf{1}}=\operatorname{diag}\left(\boldsymbol{I}_{3}, \mathbf{0}_{3}, \mathbf{0}_{2}\right), \boldsymbol{K}_{P}^{\star} \in \mathbb{R}^{3 \times 3}$ are positive definite matrices, and $\boldsymbol{e}_{E}=\boldsymbol{p}_{E}^{d}-\boldsymbol{p}_{E}, \dot{e}_{p_{R}}=\boldsymbol{v}_{R}^{n}-\boldsymbol{v}_{R}$. Notice that in (3) and (4) we neglected the dynamic couplings and Coriolis terms since a very slow motion (i.e., quasi-static condition) is required for the sought task.

Finally, the function $\sigma\left(\boldsymbol{e}_{E}\right)$ implements a particular integral action with dead-zone, applied to the interaction frame, $\mathscr{F}_{I}=\left\{O_{I}, \boldsymbol{x}_{I}, \boldsymbol{y}_{I}, \boldsymbol{z}_{I}\right\}$. While performing the desired task, namely when the sensor is in contact with the surface we define $\mathscr{F}_{I}$ such that $O_{I}$ coincides with $O_{E}$ and $z_{I}$ is directed as the normal to the surface at the point $O_{I} . \boldsymbol{x}_{I}$ and $\boldsymbol{y}_{I}$ are arbitrary. Then we denote by $\boldsymbol{R}_{I} \in S O(3)$ the rotation matrix

\footnotetext{
${ }^{2} \boldsymbol{I}_{i} \in \mathbb{R}^{i \times i}$ is the identity matrix of dimension $i \cdot \operatorname{diag}\left(\boldsymbol{A}_{1}, \boldsymbol{A}_{2}, \ldots, \boldsymbol{A}_{n}\right)$ is a block diagonal matrix where $\boldsymbol{A}_{1}, \boldsymbol{A}_{2}, \ldots, \boldsymbol{A}_{n}$ are the diagonal elements.

${ }^{3} \mathrm{~A}$ comparison of the two controllers applied to our system is available in the technical report and in the video added to the multimedia materials.
} 
describing the attitude of $\mathscr{F}_{I}$ w.r.t. $\mathscr{F}_{W}$. The integral action $\boldsymbol{\sigma}\left(\boldsymbol{e}_{E}\right)$ is then defined such as

$$
\dot{\boldsymbol{\sigma}}\left(\boldsymbol{e}_{E}\right)=\left\{\begin{array}{l}
\boldsymbol{R}_{\boldsymbol{I}}^{\top} \boldsymbol{P} \boldsymbol{R}_{\boldsymbol{I}} \boldsymbol{e}_{E} \text { if } 0<\left[\begin{array}{lll}
0 & 0 & 1
\end{array}\right] \boldsymbol{R}_{\boldsymbol{I}} \boldsymbol{e}_{E}<\boldsymbol{\varepsilon}_{I} \\
\boldsymbol{e}_{E} \text { otherwise }
\end{array},\right.
$$

where $\boldsymbol{P}=\operatorname{diag}(1,1,0)$. The integral action is needed to bring the error to zero at steady state in case of external disturbances or unknown parameters (similarly in (3)). However, along the direction of interaction $z_{I}$, the error will never go to zero, since the desired end-effector trajectory is slightly inside the pipe. Then, to avoid an infinite growth of the integral along $z_{R}$, the latter is stopped when the error along $\boldsymbol{z}_{R}$ in $\mathscr{F}_{I}$, i.e., $\left[\begin{array}{lll}0 & 0 & 1\end{array}\right] \boldsymbol{R}_{I} \boldsymbol{e}_{E}$, is below a positive threshold, $\varepsilon_{I} \in \mathbb{R}$, which is set according to the compliance of the system. A schematic representation of the controller is available in the attached technical report.

\section{EXPERIMENTAL RESULTS}

The following experiment - reached after a series of simulations and other experimental tests - is aimed to demonstrate the effectiveness of the proposed aerial manipulator system to perform inspection tasks requiring physical contact. A detailed description of the software framework and some snapshots of the experiment are available in the technical report added to the multimedia materials.

The goal of the experiment is to identify and localize a weld on a portion of metallic pipe (see Figs. 1 and 2) of length $0.5[\mathrm{~m}]$ and diameter $0.4[\mathrm{~m}]$, using an EC probe. The inspection is performed on a section of the pipe by means of a raster scan path passing over the weld 4 times. Giving the set of way-points as input to the proposed motion planner, we obtain the desired end-effector trajectory and the nominal trajectories of the robot DoFs shown in Figs. 3 and 4 , respectively. The contact-inspection trajectory has a duration of $110[\mathrm{~s}]$ (only the contact phase) and is planned in around $9[\mathrm{~s}]$ on a standard laptop.

The overall experiment is composed of three parts: i) approach to the pipe (arm folded and sensor not in contact), ii) contact-based inspection, and iii) departure from the pipe. Here, we focus our analysis on the most interesting part ii). For this part, Figs. 3, 4 and 5 show the behavior of the robot and the sensor data, from right before contact (time $25[\mathrm{~s}]$ ) to right after contact (once the inspection is over, time 144 [s]).

From Figs. 3 and 4, one can appreciate the accuracy of the robot to track the desired end-effector trajectory keeping always the contact (confirmed by the value of $w_{l}$ ). This is done despite the presence of many uncertainties, elasticity, frictions, and no force feedback. The presence of errors in the kinematic model is confirmed by the non-zero error between the nominal and actual pose of the aerial vehicle.

Notice that once the robot gets in contact with the surface, the (unknown) interaction force exerts an extra torque on the aerial vehicle, which implies an initial orientation error. This error goes to zero in few seconds as a result of the integral term in (3), which can act effectively and independently from the position control. During the transient, this inaccuracy

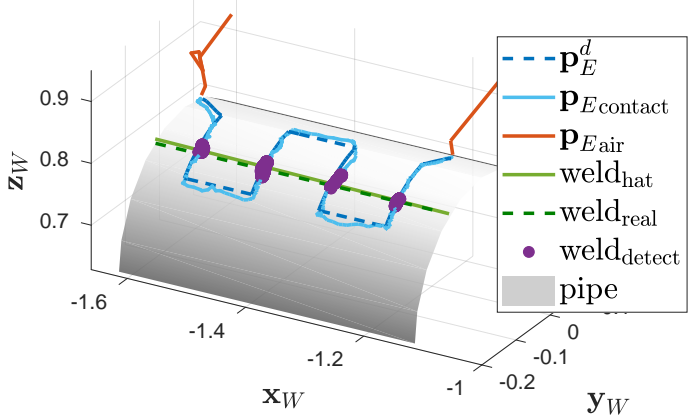

Fig. 3: Representation of the desired and actual trajectories of the end-effector over the pipe. The purple dots highlight the points in which the weld is detected.
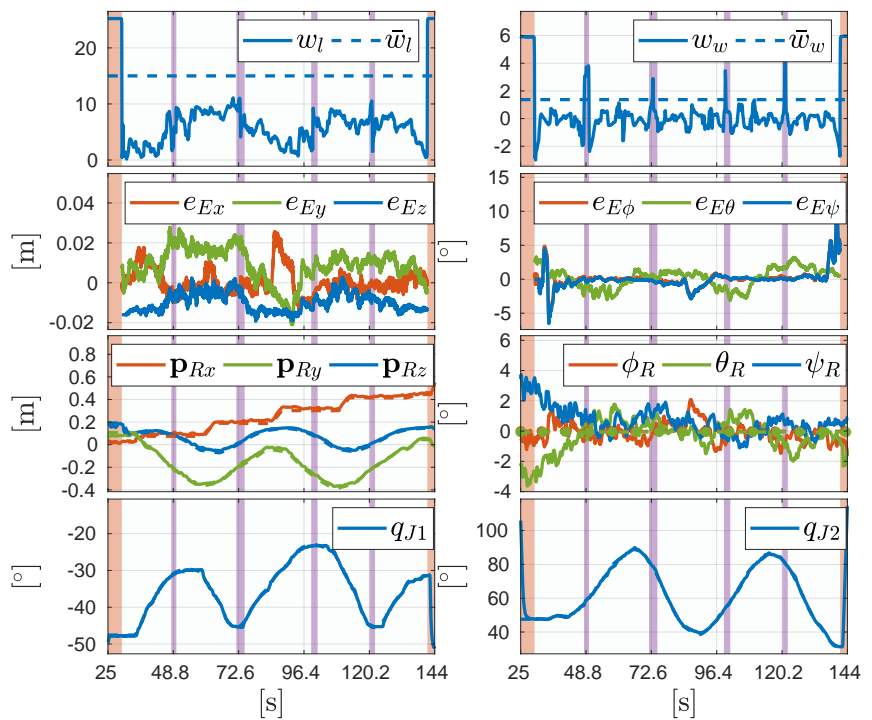

Fig. 4: Evolution of the main variables. The dashed lines in the last four plots represent the nominal trajectories given by the motion planner. For convenience, the aerial vehicle orientation is displayed by the Euler-angles $\left(\psi_{R}, \theta_{R}, \phi_{R}\right)$ following the convention Z-Y-X.

induces a small error in the end-effector orientation too. The latter is defined by the vector $e_{E \eta}=\left[\begin{array}{lll}e_{E \phi} & e_{E \theta} & e_{E \psi}\end{array}\right]^{\top}$ where $e_{E \phi}=\phi_{E}^{d}-\phi_{E}$. Analogously for $e_{E \theta}$ and $e_{E \psi}$. For display purposes we show $\left(\psi_{E}, \theta_{E}, \phi_{E}\right)$ as the Euler-angles describing the orientation of $\mathscr{F}_{E}$, following the convention $\mathrm{Z}-\mathrm{Y}-\mathrm{X}$. The end-effector pose error always respects the task requirements. $e_{E \eta}$ shows the deviation of the end-effector orientation from being perpendicular to the surface. The perpendicularity of end-effector is further shown in the attached video.

Fig. 5 shows the raw signal $w(t)$ coming from the EC sensor. It is interesting to notice its evolution when the probe passes from air to contact and over the weld. From $w(t)$, applying the mentioned PCA method, we can retrieve the more informative signals $w_{l}$ and $w_{w}$ (shown in Fig. 4). The contact phase can be identified by looking at when $w_{l}<\bar{w}_{l}=$ 15. On the other hand, looking at $w_{w}$ for $w_{w}>\bar{w}_{w}=1.4$ and $w_{l}<\bar{w}_{l}$, we can identify when the probe is in contact with the weld. The thresholds $\bar{w}_{l}$ and $\bar{w}_{w}$ were identified in a preliminary calibration phase. In all the plots, we highlight 

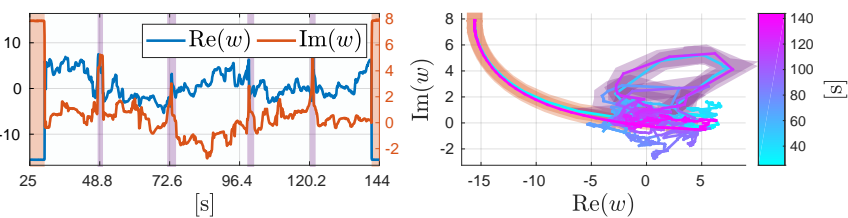

Fig. 5: Acquired raw data $w$, showing its real and imaginary parts and its evolution in $\mathbb{C}$. On the right, the color represents the time.

the no-contact and contact-with-weld phases with red and purple colors, respectively.

Combining $w_{w}$ with the measured position of the probe, we retrieved an estimation of the weld position all along the surface, using simple linear regression. The estimated and real weld positions, defined by weld $\mathrm{real}_{\text {rea }}$ and weld $\mathrm{d}_{\text {hat }}$, are shown in Fig. 3 by green solid and dashed lines, respectively.

In view of a future integration with a GPS+vision-based system for outdoor experimentation, we tested the proposed robotic system with degraded MoCap measurements. We reduced the state-update frequency down to $30[\mathrm{~Hz}]$ and added a white noise signal of standard deviation equal to $1[\mathrm{~cm}]$ and $2\left[^{\circ}\right]$ to the position and orientation components of the MoCap measurement (comparable to an outdoor localization system based on differential GPS and cameras). Under this condition, the tracking errors obviously increase, but remain sufficiently small to perform the desired task, preserving the contact between the sensor and the pipe surface. We tested the system also in the presence of wind, produced by a fan blowing air on the robot at a speed of around $6[\mathrm{~m} / \mathrm{s}]$. This disturbance has almost no effect on the quality of the results. The corresponding results are presented in the technical report and videos added to the multimedia materials.

\section{CONCLUSIONS}

In this work, we considered the challenging physical interaction task of pushing while sliding on curved surfaces with an aerial manipulator, going beyond the simpler pick\&place and pull/push tasks. We proposed one of the first aerial manipulator systems capable to perform such a task in the context of a real and relevant application: EC inspection of metallic pipes. The model inaccuracy, the lack of force feedback, as well as the presence of elasticity in the arm structure required the design of a selective and displaced PID-based controller inspired by the control of manipulators with elastic joints. Together with a suitable task-constrained motion planning method, we demonstrated the capability of the robot to perform the task, detecting and mapping the weld accurately. The robotic system will be employed in the near feature also for the detection of cracks along the weld. Another natural follow-up of this work will be the integration with an onboard localization system to perform the task outdoor in a real industrial plant.

\section{REFERENCES}

[1] J. García-Martn, J. Gómez-Gil, and E. Vázquez-Sánchez, "Nondestructive techniques based on eddy current testing," Sensors, vol. 11, no. 3, pp. 2525-2565, 2011.
[2] E. Gasparin, G. Santi, and A. Nussbaumer, "Eddy current crack monitoring system for structural health monitoring (SHM) applications," in 68th Int. Institute for Welding (IIW) Annual Assembly and Int. Conf., Helsinki, Finland, July 2018, pp. 1084-1090.

[3] J. V. Miro, D. Hunt, N. Ulapane, and M. Behrens, "Towards automatic robotic NDT dense mapping for pipeline integrity inspection," in 11th Int. Conf. on Field and Service Robotics, Zurich, Switzerland, Sep. 2017, pp. 319-333.

[4] A. Ollero, G. Heredia, A. Franchi, G. Antonelli, K. Kondak, A. Sanfeliu, A. Viguria, J. R. Martinez-de Dios, F. Pierri, J. Cortés, A. Santamaria-Navarro, M. A. Trujillo, R. Balachandran, J. AndradeCetto, and A. Rodriguez, "The AEROARMS project: Aerial robots with advanced manipulation capabilities for inspection and maintenance," IEEE Robotics \& Automation Magazine, Special Issue on Floating-base (Aerial and Underwater) Manipulation, vol. 25, no. 4, pp. 12-23, 2018

[5] F. Ruggiero, M. A. Trujillo, R. Cano, H. Ascorbe, A. Viguria, C. Perz, V. Lippiello, A. Ollero, and B. Siciliano, "A multilayer control for multirotor UAVs equipped with a servo robot arm," in 2015 IEEE Int. Conf. on Robotics and Automation, Seattle, WA, May 2015, pp. 4014-4020.

[6] M. Kamel, S. Comari, and R. Siegwart, "Full-body multi-objective controller for aerial manipulation," in 24th Mediterranean Conf. on Control and Automation, Athens, Greece, Aug. 2016, pp. 659-664.

[7] M. Ryll, G. Muscio, F. Pierri, E. Cataldi, G. Antonelli, F. Caccavale, and A. Franchi, "6D physical interaction with a fully actuated aerial robot," in 2017 IEEE Int. Conf. on Robotics and Automation, Singapore, May 2017, pp. 5190-5195.

[8] M. Tognon, B. Yüksel, G. Buondonno, and A. Franchi, "Dynamic decentralized control for protocentric aerial manipulators," in 2017 IEEE Int. Conf. on Robotics and Automation, Singapore, May 2017, pp. 6375-6380.

[9] M. Ryll, D. Bicego, and A. Franchi, "A truly redundant aerial manipulator exploiting a multi-directional thrust base," in 2018 IFAC Symp. on Robot Control, Budapest, Hungary, Aug. 2018.

[10] K. Alexis, G. Darivianakis, M. Burri, and R. Siegwart, "Aerial robotic contact-based inspection: planning and control," Autonomous Robots, vol. 40, no. 4, pp. 631-655, 2016.

[11] M. Fumagalli, R. Naldi, A. Macchelli, F. Forte, A. Q. Keemink, S. Stramigioli, R. Carloni, and L. Marconi, "Physical interaction with the environment," IEEE Robotics \& Automation Magazine, vol. 21 , no. $3,2014$.

[12] I. Jolliffe, "Principal component analysis," in International Encyclopedia of Statistical Science. Springer, 2011, pp. 1094-1096.

[13] H. Nguyen, C. Ha, and D. Lee, "Mechanics, control and internal dynamics of quadrotor tool operation," Automatica, vol. 61, pp. 289$301,2015$.

[14] S. Park, J. J. Her, J. Kim, and D. Lee, "Design, modeling and control of omni-directional aerial robot," in 2016 IEEE/RSJ Int. Conf. on Intelligent Robots and Systems, Daejeon, South Korea, 2016, pp. 1570-1575.

[15] M. Ryll, D. Bicego, and A. Franchi, "Modeling and control of FASTHex: a fully-actuated by synchronized-tilting hexarotor," in 2016 IEEE/RSJ Int. Conf. on Intelligent Robots and Systems, Daejeon, South Korea, Oct. 2016, pp. 1689-1694.

[16] M. Tognon, E. Cataldi, H. Tello Chavez, G. Antonelli, J. Cortés, and A. Franchi, "Control-aware motion planning for task-constrained aerial manipulation," IEEE Robotics and Automation Letters, Special Issue on Aerial Manipulation, vol. 3, no. 3, pp. 2478-2484, 2018.

[17] N. Staub, D. Bicego, Q. Sablé, V. Arellano-Quintana, S. Mishra, and A. Franchi, "Towards a flying assistant paradigm: the OTHex," in 2018 IEEE Int. Conf. on Robotics and Automation, Brisbane, Australia, May 2018, pp. 6997-7002.

[18] R. Cano, C. Perez, F. Pruaño, A. Ollero, and G. Heredia, "Mechanical design of a 6-dof aerial manipulator for assembling bar structures using uavs," in 2nd RED-UAS 2013 workshop on research, education and development of unmanned aerial systems, 2014.

[19] C. C. de Wit, B. Siciliano, and G. Bastin, "Motion and force control," in Theory of robot control. Springer, 1996, ch. 4, pp. 141-175.

[20] A. De Luca and W. Book, "Robots with flexible elements," in Springer Handbook of Robotics, B. Siciliano, O. Khatib. Springer, 2008, ch. 13 , pp. 287-317. 


\title{
Additional Experiments and Descriptions
}

\author{
Technical report of: \\ "A Truly Redundant Aerial Manipulator System \\ with Application to Push-and-Slide Inspection in Industrial Plants" \\ IEEE Robotics and Automation Letters
}

2019

\begin{abstract}
Marco Tognon ${ }^{1}$, Hermes A. Tello Chávez ${ }^{1}$, Enrico Gasparin ${ }^{2}$, Quentin Sablé ${ }^{1}$, Davide Bicego ${ }^{1}$, Anthony Mallet ${ }^{1}$, Marc Lany ${ }^{2}$, Gilles Santi ${ }^{2}$, Bernard Revaz ${ }^{2}$, Juan Cortés ${ }^{1}$, Antonio Franchi ${ }^{1}$
\end{abstract}

\author{
Abstract-This document is a technical attachment to [1] \\ containing additional details on the proposed robotic system, \\ and extra experimental results.

\section{How TO Cite THIS WORK} \\ This technical report is accompanying our IEEE Robotics \\ and Automation Letters paper [1]. If you wish to reference \\ this work, please cite this paper as follows:

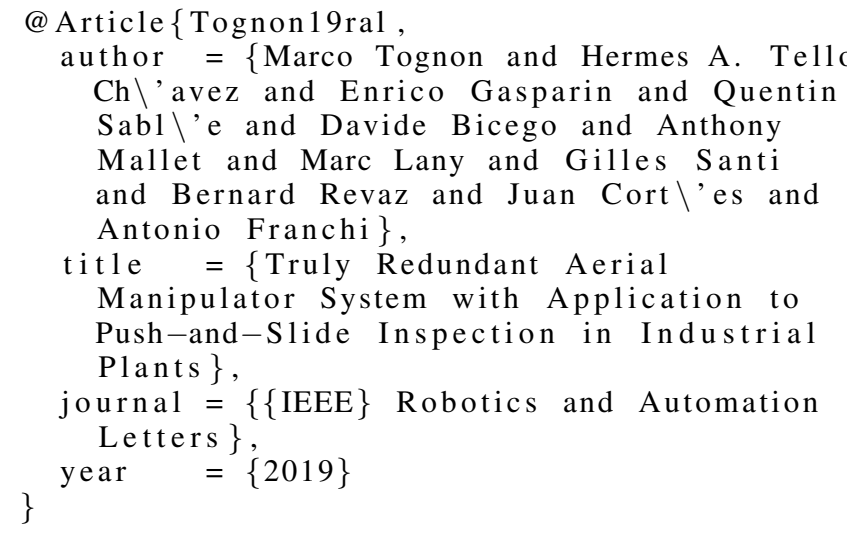

\section{AERIAL MANIPULATOR}

The main physical parameters of the whole aerial manipulator are reported in Tab. I.

\section{A. Flexible 2 d.o.f. arm}

In [1] we mention that the employed extreme lightweight arm exhibits some flexibility at the joints and structure levels, due to its reduced rigidity. Indeed, the tensegrity structure, based on carbon fiber bars and 3D printed parts, deforms when subject to external forces. Similarly, also the belt that distributes the actuation of the second motor to the

\begin{tabular}{|c|c|c|c|c|c|}
\hline \multicolumn{2}{|l|}{ OTHex } & \multicolumn{2}{|l|}{ 2-DoFs arm } & \multicolumn{2}{|c|}{ Sensor+holder } \\
\hline mass $[\mathrm{kg}]$ & 1.6 & mass $[\mathrm{kg}]$ & 0.45 & mass $[\mathrm{kg}]$ & 0.2 \\
\hline diameter $[\mathrm{m}]$ & 1.2 & length link $1[\mathrm{~m}]$ & 0.29 & length $[\mathrm{m}]$ & 0.13 \\
\hline height $[\mathrm{m}]$ & 0.3 & length link $2[\mathrm{~m}]$ & 0.25 & & \\
\hline
\end{tabular}

TABLE I: Physical parameters of the aerial manipulator.

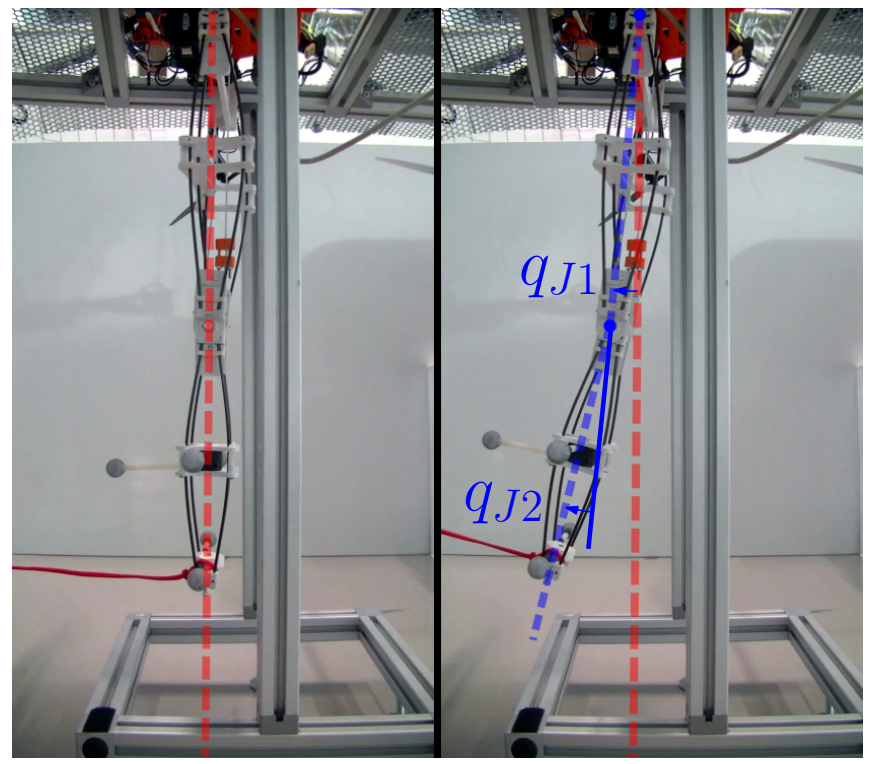

Fig. 1: On the left the arm is fully stretched without external forces acting on the tip. On the right, a force of $4.5[\mathrm{~N}]$ is applied to the tip of the arm. Comparing the two images one can appreciate the flexibility of the arm at the joint and structure level.

second link deforms when subject to external forces, due to backlashes and the proper elasticity of the belt.

To assess the flexibility of the arm we performed additional tests in which we fixed the aerial platform in a horizontal configuration. The arm is placed in a stretched down configuration (zero relative joint angles) and the motors are blocked in order to keep the configuration. In such condition, we applied forces with different intensities to the tip of the arm recording the deviation of the joints angles

\begin{tabular}{ccc}
\hline Force intensity & $q_{J 1}$ & $q_{J 2}$ \\
\hline $2.0[\mathrm{~N}]$ & $2.6\left[^{\circ}\right]$ & $3.7\left[^{\circ}\right]$ \\
$4.5[\mathrm{~N}]$ & $4.3\left[^{\circ}\right]$ & $8.6\left[^{\circ}\right]$ \\
\hline
\end{tabular}

TABLE II: Deviation of the joint angles from the zero value (stretched arm) for two external forces with different intensities applied to the tip of the arm. 


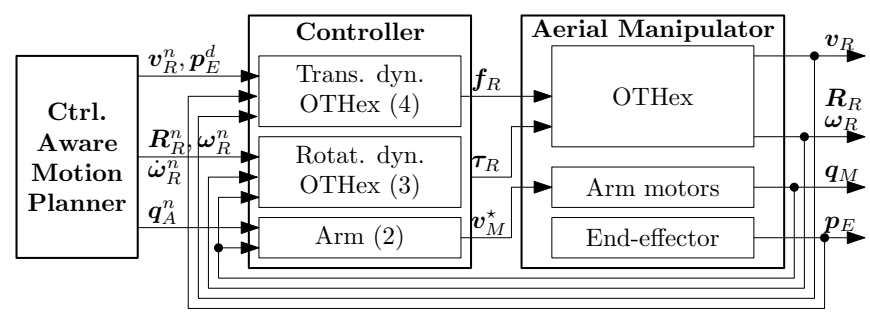

Fig. 2: Schematic representation of the controller.

from the zero value. Table II reports the maximum deviation of the arm joints for two forces of increasing intensity. Figure 1 shows the deviation of the arm when an external force of $4.5[\mathrm{~N}]$ is applied to the tip. We remark that those joint deviations are due to deformations of the structure and not to the actual motion of the motors. Therefore, they cannot be measured by solely the motor encoders.

\section{Control Method}

Figure 2 visually describes the controller. Summarizing, a direct measure or an estimation of the following variables is needed: $\boldsymbol{v}_{R}, \boldsymbol{R}_{R}, \boldsymbol{\omega}_{R}, \boldsymbol{q}_{M}$ (or equivalently $\boldsymbol{q}_{A}$ ) and $\boldsymbol{p}_{E}$.

\section{A. Dislocated V.S. End-effector PD controller}

In this section we provide the results obtained comparing the 'dislocated' controller proposed in [1] and a standard PD controller with entire feedback from the end-effector, i.e. replacing $\dot{e}_{p_{R}}$ with $\dot{e}_{E}=\boldsymbol{v}_{E}^{d}-\boldsymbol{v}_{E}$ in (4) of [1], obtaining:

$$
\begin{aligned}
\boldsymbol{f}_{R}= & \boldsymbol{R}_{R}^{\top}\left(\boldsymbol{g}_{1}(\boldsymbol{q})+M_{11} \dot{\boldsymbol{v}}_{R}^{n}+\boldsymbol{K}_{P}^{D} \dot{\boldsymbol{e}}_{E}+\right. \\
& \left.+\boldsymbol{K}_{P}^{P} \boldsymbol{e}_{E}+\boldsymbol{K}_{P}^{I} \boldsymbol{\sigma}\left(\boldsymbol{e}_{E}\right)\right) .
\end{aligned}
$$

The comparison has been done requiring the robot to keep the end-effector in a desired position and switching between the two controllers to observe the different performance. Figure 3 shows the norm of the position, velocity and acceleration errors of both the aerial vehicle (w.r.t. the nominal position) and the end-effector (w.r.t. the desired position), on the left and right columns, respectively. The yellow areas represent the time intervals in which controller (1) is used, while the other areas the dislocated controller proposed in the paper (equation (4) of [1]) is used. One can immediately notice that, as soon as the controller (1) is activated, the errors start to diverge bringing the system to almost instability. In fact, especially in the second interval, after few seconds we had to switch the controller back to our proposed dislocated controller in order to avoid a crash. The latter is able to safely bring the system to stability. A video of the experiment is attached to the manuscript [1].

\section{SOFTWARE ARCHITECTURE}

The software developed for the presented aerial manipulator comprises many independent and interconnected components. The resulting software architecture is made of only in-house open source software.

At the lowest level, the OTHex runs a custom software on Mikrokopter brushless controllers (8bit Atmega MCU). The
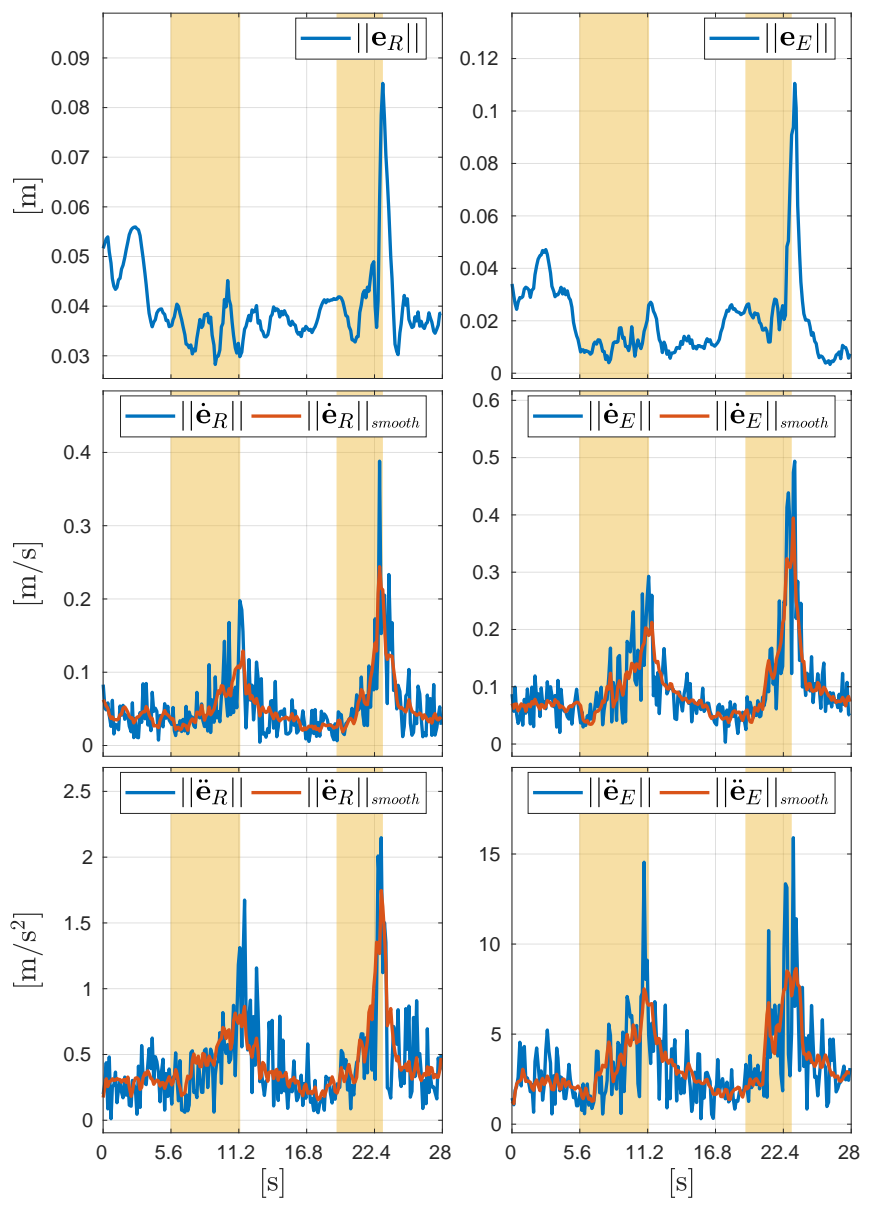

Fig. 3: Comparison between our proposed dislocated PD controller (equation (4) of [1]) and a standard end-effector PD controller (equation (1)). On the left the norm of the position, velocity and acceleration errors of the aerial vehicle w.r.t. the nominal values, defined by the vectors $\boldsymbol{e}_{\boldsymbol{p}_{R}}, \dot{\boldsymbol{e}}_{\boldsymbol{p}_{R}}$ and $\ddot{\boldsymbol{e}}_{\boldsymbol{p}_{R}}$, respectively. On the right the norm of the position, velocity and acceleration errors of the end-effector w.r.t. the desired values, defined by the vectors $\boldsymbol{e}_{E}, \dot{\boldsymbol{e}}_{E}$ and $\ddot{e}_{E}$, respectively. For the velocity and acceleration errors we plotted, as a red line, a smoothed version of each quantity, also indicated with the subscript $\star_{\text {smooth }}$.

code implements a closed loop, precise propeller velocity controller [2] and communicates via an I2C bus with a Mikrokopter flight controller. The precise control of the spinning velocity is very important for an accurate actuation of the desired wrench. The flight controller acts as a proxy between the brushless motors and the higher software levels. It also exports the raw, analog 6D IMU readings to the higher software levels. Both components run at $1 \mathrm{kHz}$.

The higher software layer is made of a variable number of independent components, including:

1) A UAV driver component interfacing with the flight controller through a USB UART link. It sends the desired propeller velocities to the hardware and exports actual measurements, IMU, current consumption etc.

2) A Dynamixel motor driver component interfacing with the 2-DoFs arm. It sends the desired velocity to each motor 
and reads its actual velocity and position.

3) A UKF based state estimation component, running at $1 \mathrm{kHz}$, that fuses data from IMU, motion capture system or any other source.

4) A motion capture component reading multiple rigid bodies (namely OTHex and end-effector) position and orientation from an Optitrack system, from $30[\mathrm{~Hz}]$ to $100[\mathrm{~Hz}]$.

Most of those components have been developed in $\mathrm{C}++$ and can run either onboard or on an off-board desktop PC. The actual control components (attitude, position, and physical interaction) are currently developed in Matlab/Simulink and run on a ground $\mathrm{PC}$ at $500[\mathrm{~Hz}]$. Those components will be soon implemented in $\mathrm{C}++$ too, so that the whole demonstration can run on an embedded computer.

As for most of the robotics software at LAAS-CNRS, software components have been developed using GenoM3 [3], a code generator and formal software component description language that allows assembling middleware-independent components in a modular system. Most of this software is available on the openrobots repository at https://git. openrobots.org/projects/telekyb3

\section{ADDITIONAL EXPERIMENTS}

In view of a future integration with a vision system for outdoor experimentation, we tested the proposed robotic system with degraded MoCap measurements and the presence of a wind gust. In Fig. 4 we show few images of one experiment.

\section{A. Degraded MoCap measurements}

First, we report the tracking performance of the proposed robotic system when the update rate of the state is reduced. In particular, in Fig. 5 we report the experimental results during the interaction phase, when the MoCap frequency is set to only $30[\mathrm{~Hz}]$. Comparing those results with the ones obtained with the MoCap running at $100[\mathrm{~Hz}]$ (see Fig. 4 of [1]) we can conclude that there are no substantial differences.

Then, we further degraded the MoCap measurements reducing its accuracy. In details, we added a white noise of standard deviation $0.01[\mathrm{~m}]$ and $2\left[^{\circ}\right]$ in the position and orientation MoCap measurements, respectively. This level of precision could be comparable to a system with onboard sensors like GPS and/or cameras [4], [5]. The experimental results are shown in Fig. 6 where we plotted the end-effector errors and the configuration of the system with respect to the nominal value. Also in this case, the tracking errors obviously increase, but remain sufficiently small to perform the desired task. A video of the corresponding experiment can be found in the multimedia materials attached to [1].

\section{B. External disturbance: wind}

In this experiment a domestic fan blows air toward the aerial manipulator. The air has a speed of around $6[\mathrm{~m} / \mathrm{s}]$. We configured the fan in order to blow air on the robot from the worst direction, i.e., the wind gust pushes the robot away from the pipe. In this configuration the robot could lose contact with the surface. In Fig. 7 we report the corresponding results. Also in this case, comparing the results with the one without the wind gust (see Fig. 4 of [1]) we cannot spot any relevant differences. We can conclude that the proposed robotic system is also robust to sufficiently bounded external disturbances.

\section{REFERENCES}

[1] M. Tognon, H. A. Tello Chávez, E. Gasparin, Q. Sablé, D. Bicego, A. Mallet, M. Lany, G. Santi, B. Revaz, J. Cortés, and A. Franchi, "A truly redundant aerial manipulator system with application to push-andslide inspection in industrial plants," IEEE Robotics and Automation Letters, 2019.

[2] A. Franchi and A. Mallet, "Adaptive closed-loop speed control of BLDC motors with applications to multi-rotor aerial vehicles," in 2017 IEEE Int. Conf. on Robotics and Automation, Singapore, May 2017, pp. 52035208.

[3] A. Mallet, C. Pasteur, M. Herrb, S. Lemaignan, and F. Ingrand, "Genom3: Building middleware-independent robotic components," 2010 IEEE International Conference on Robotics and Automation, pp. 4627-4632, 2010.

[4] R. Mur-Artal, J. M. M. Montiel, and J. D. Tardos, "Orb-slam: a versatile and accurate monocular slam system," IEEE Transactions on Robotics, vol. 31, no. 5, pp. 1147-1163, 2015.

[5] C. Forster, M. Pizzoli, and D. Scaramuzza, "Svo: Fast semi-direct monocular visual odometry," in Robotics and Automation (ICRA), 2014 IEEE International Conference on. IEEE, 2014, pp. 15-22. 

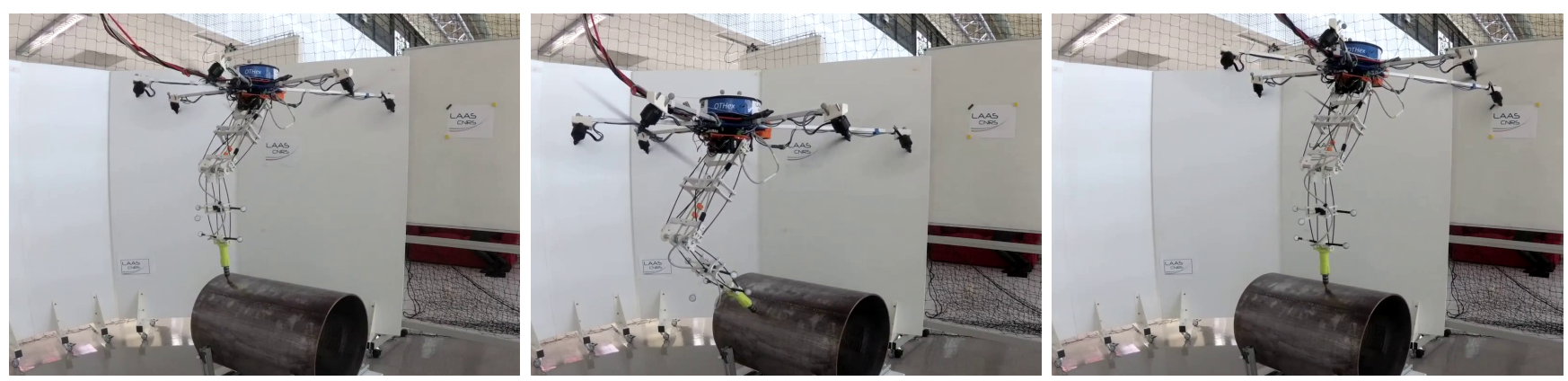

Fig. 4: A series of three images during the inspection of the pipe.
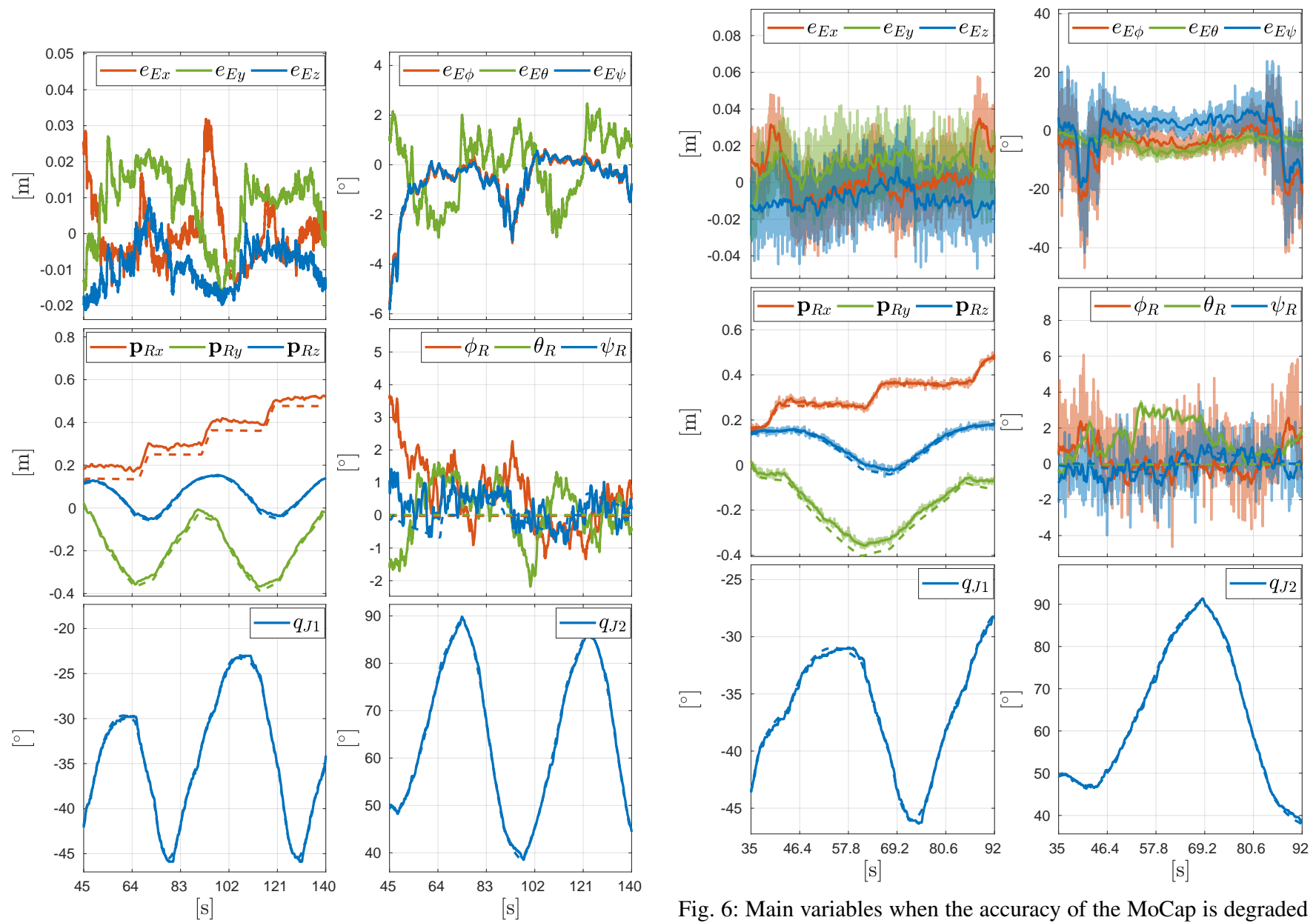

Fig. 5: Main variables when the MoCap frequency is reduced to $30[\mathrm{~Hz}]$.

Fig. 6: Main variables when the accuracy of the MoCap is degraded with the addition of white noise. In opaque there are the variables measured by the mocap while in solid the real values. We remark that the control feedback is done with the estimated state based on the noisy measurements. 

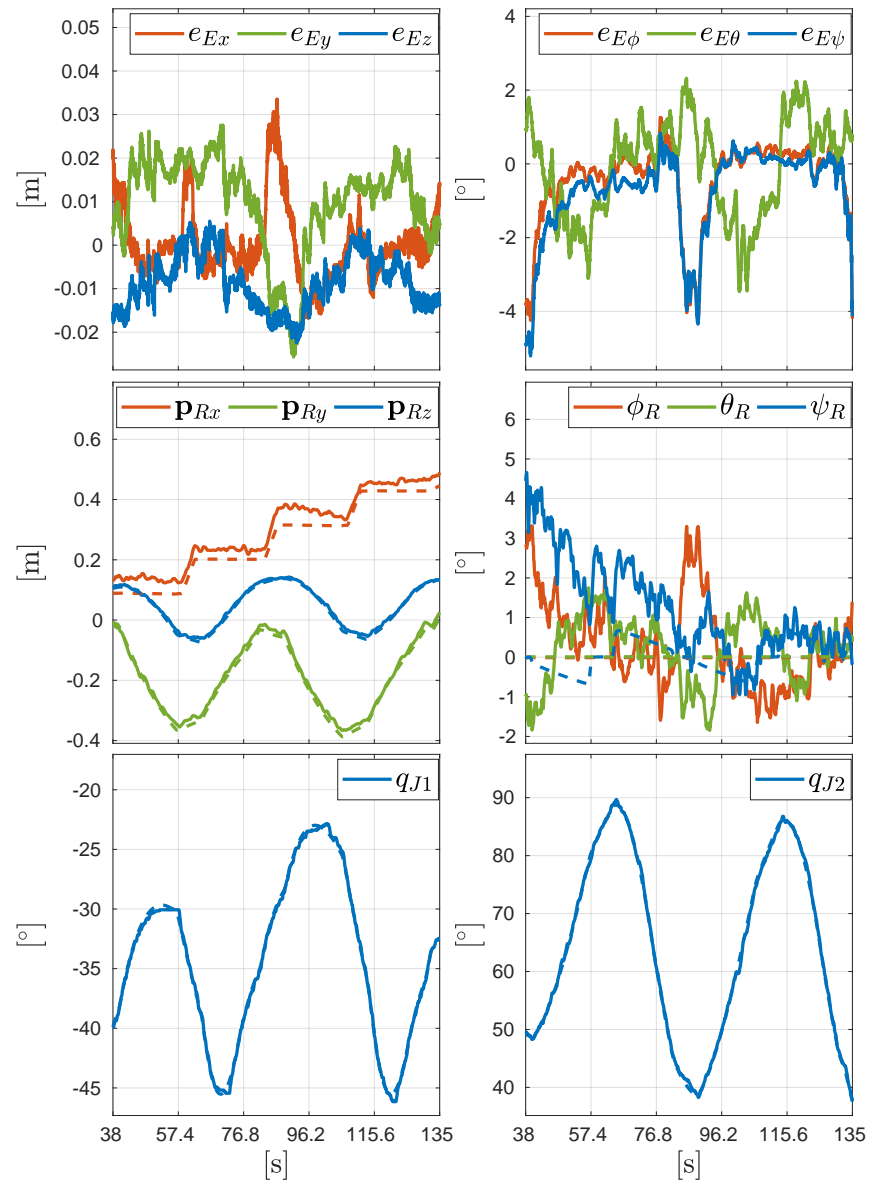

Fig. 7: Main variables when the aerial manipulator is subjected to an external force, produce by a wind gust, pushing it away from the pipe. 